\title{
AN INDUCTION LINAC DRIVER FOR A 0.44 MJ HEAVY-ION DIRECT DRIVE TARGET
}

\author{
by \\ P.A. Seidl, E.P. Lee, R.O. Bangerter, A. Faltens \\ Lawrence Berkeley National Laboratory (on behalf of U.S. HIFS-VNL) \\ 1 Cyclotron Road, Berkeley, CA 94720,
}

Accelerator Fusion Research Division

Ernest Orlando Lawrence Berkeley National Laboratory

University of California

Berkeley, California 94720

February 2010

This work was supported by the Director, Office of Science, Office of Fusion Energy Sciences, of the U.S. Department of Energy under Contract No. DE-AC0205CH11231. 
HIFAN - 1768

LBNL

\author{
An Induction Linac Driver For A 0.44 MJ Heavy-Ion Direct Drive \\ Target \\ February 8, 2010 \\ P.A. Seidl, E.P. Lee, R.O. Bangerter and A. Faltens \\ $L B N L$
}

\begin{abstract}
$\underline{\text { Abstract }}$
The conceptual design of a heavy ion fusion driver system is described, including all major components. Particular issues emerging from this exercise are identified and discussed. The most important conclusion of our study is that due to stringent requirements on ion pulse phase space, we are unable to find a credible accelerator design that meets the requirements of the example target. Either the target design must be modified to accept larger ion ranges and larger focal spot sizes, or we must consider other target options.
\end{abstract}

\title{
1 Introduction and Summary
}

Following basic research, the current HIF development path envisions a sequence of three major facilities. The first is an integrated research experiment (IRE) which tests all beam manipulations at or near driver scale in key beam parameters. The IRE will also be capable of addressing the most pressing HIF target issues at target temperatures exceeding $100 \mathrm{eV}$ (within a factor of a few of the temperatures produced by a full-scale driver). The IRE will be followed by an engineering test facility (ETF) which would include all elements of the reactor-driver interface, ignite fusion targets at the pulse repetition rate required for a power plant, and produce of the order of $100 \mathrm{MW}$ of fusion power [Sn-02]. The conceptual facility outlined in this report is an ETF facility. The final step in the development path preceding commercial deployment is a demonstration power plant (DEMO) producing of the order of $1 \mathrm{GW}$ of electrical power. It is generally assumed that the IRE and ETF should use design strategies that clearly and relatively simply extrapolate to the DEMO driver. Indeed, it may be possible to upgrade the IRE accelerator to drive the ETF or the ETF accelerator to drive the DEMO.

Given a specific target design, the task of the accelerator and transport system is to supply the requisite number of beams with the required spatial and temporal distribution around the reactor chamber and fusion target. The chamber design is a separate discipline, with the tasks of providing a neutron, blast, and x-ray absorbing "wall" with high longevity. Additional shielding is required in front of and inside of the final focusing magnets to protect the magnets winding. The accelerator itself is usually hundreds of meters away from the chamber. Within the final transport lines, the beam bunches are caused to shorten by a factor of 150-1000, thereby increasing the beam power by that same factor to the level required by the fusion capsule.

In previous target and multi-GeV driver designs, given the phase space and brightness requirements from the target and final focusing system, we found that at the other end of the 
accelerator, that is, at the source, heavy ion beams can be generated with a substantial safety factor. This is essential because part of the design is deciding how to apportion the safety factor among various parts of the accelerator.

There are a large number of target designs and accelerator architectures that might be used for the ETF. In this paper we consider only one specific target design, namely the 0.44-MJ direct drive target of Perkins and Hay [Lo-08, Lo-09]. Subsequent studies will use other target designs. The principal motivations for using the target of Perkins and Hay in this study are its relatively low driver energy requirement, its relatively simple construction, and its simple chemical and activation characteristics. In particular, initially it seemed plausible that the low energy requirement might translate into low driver cost.

The range of the ions required for both the foot and the main pulse of the Perkins and Hay target is very small compared those of most other target designs. Small range, for a given ion mass, implies low kinetic energy. We know a priori that low kinetic energy also makes it difficult to meet phase-space and transport requirements unless one uses a very large number of beams. Some of the most challenging parts of an accelerator have been the complex of multiple beam sources, matching, and transport at low energies, with the transport becoming easier as energy is increased because the half periods of the focusing lattice get longer, and the induction linac cores get smaller because of decreasing pulse duration. Electrostatic quadrupoles (ESQs) compete favorably with other beam transport options at low kinetic energy, and have been the choice for low energy beam transport experiments [Fe-89] of 82 quadrupoles in a single beam channel and 70 multiple beam quadrupole arrays in MBE-4. They scale well to arrays containing many, small beams. Thus, an all-ESQ accelerator is intriguing from an economic and short development path standpoint for the relatively low ion energies assumed here $(500 \mathrm{MeV}$ and 50 $\mathrm{MeV} \mathrm{Rb}^{+}$). Based on these considerations we chose to study accelerator architectures using only ESQ focusing. It can be argued that the use of ESQs only might obviate the need to develop arrays of magnetic quadrupoles. Thus, if it were possible to use ESQs only, it would endow this approach with a programmatic advantage. Moreover, the use of ESQs reduces concerns about the effects of electrons (usually unwanted) on beam dynamics because the electric fields sweep the unwanted electrons out of the beam.

Unfortunately, ESQs cannot focus strongly enough to confine the beam as it compresses longitudinally after exiting the accelerator. It is therefore necessary to use magnetic quadrupole arrays in the compression lines. Nevertheless, it is probably easier to develop magnetic arrays for use outside the accelerator than for use inside the accelerator so there might still be some programmatic advantage.

The most important conclusion of our study is that due to stringent requirements on ion pulse phase space, we are unable to find a credible accelerator design that meets the requirements of the example target. Either the target design must be modified to accept larger ion ranges and/or larger focal spot sizes, particularly for the foot pulse, or we must consider other target options. In addition, the current target design assumes spherical illumination. It would be highly advantageous if the target could be modified to use illumination configurations that are more compatible with liquid wall protection, as discussed in Section 4. 
This report is divided into sections covering various aspects of the driver, followed by a section on our findings and suggestions for future directions. This report is not based on a system optimization such as generated by the LIACEP program developed in the 1980's [Ho-88] or the IBEAM program developed in the 1990's and applied to the "robust point design" (RPD) for a heavy ion fusion power plant based on indirectly driven targets [Yu-03]. Rather, this is an estimate of the feasibility of developing a driver for the Perkins and Hay directly driven target. It is based primarily on ion-pulse phase space considerations, and previously documented HIF driver injection, transport, induction acceleration, drift compression and final focusing R\&D.

\section{Table of Contents}

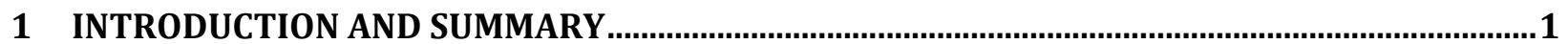

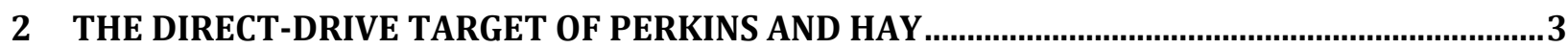

3 PHASE SPACE, NUMBER OF BEAMS AND GROUPING OF BEAMS ...............................................

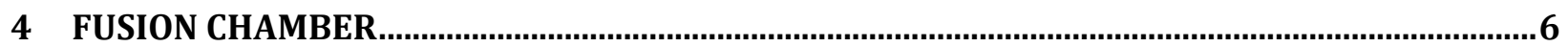

5 DRIVER SYSTEM

6 BEAM SOURCE AND INJECTOR

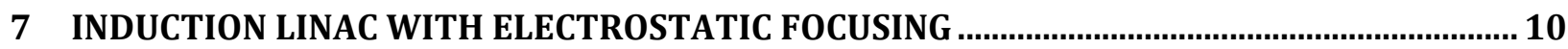

8 LINAC SCALING AND INDUCTION CORE REQUIREMENTS ….................................................... 13

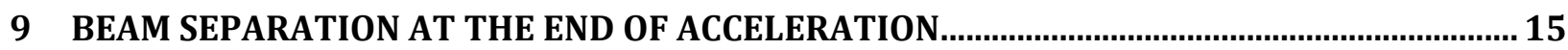

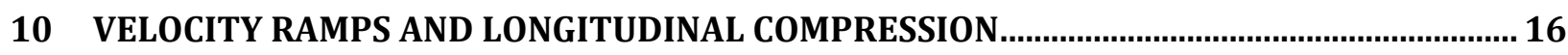

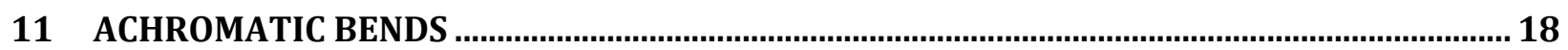

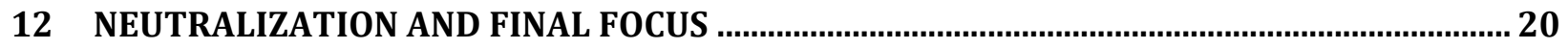

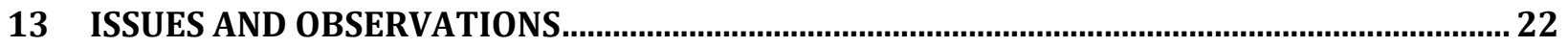

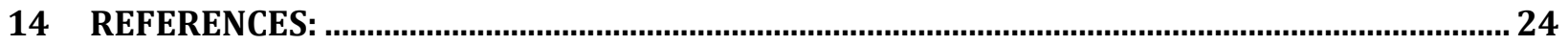

\section{The Direct-Drive Target Of Perkins and Hay}

The target is a spherical shell, comprised of a 50-50 mixture of deuterium and tritium. The outer frozen shell thickness is $0.47 \mathrm{~mm}$, the outer shell radius is $1.17 \mathrm{~mm}$ and the initial aspect ratio (shell radius to shell thickness $\approx 2$ ) is relatively low. The gain is approximately 44, according to one-dimensional Hydra and Lasnex simulations.

In the context of this report, there are several important comments about the pulse shape for this target, shown in Figure 1. In order to achieve high target gain, it is necessary to compress most of the fuel to many times its initial density of about $0.26 \mathrm{~g} / \mathrm{cc}$. Unless the fuel is at sufficiently low temperature that it is nearly Fermi-degenerate, the compression energy is excessive. If the initial power is too high, strong shock waves are produced that can heat the fuel to temperatures well beyond the Fermi-degenerate state. Therefore the power pulse shapes for nearly all targets have the general characteristics shown in Figure 1, namely a low-power foot that increases gradually, or in steps, to the peak power at the end of the pulse. For indirectly driven targets, the 
ratio of peak power to foot power is usually less than the corresponding ratio for direct drive because some of the energy penalties associated with indirect drive can be paid early in time. The lines showing two different ion kinetic energies, one for the foot and one for the main pulse, is somewhat atypical. Some target designs use the same ion kinetic energy for the foot and the main pulses. Most targets that do not use the same kinetic energy for both usually have kinetic energies that differ by less than the factor of 10 required for our representative target. This large ratio is one of the considerations that make it difficult to find an attractive driver option for this type of target.

\section{Phase Space, Number of Beams and Grouping of Beams}

The number of beams required to drive the target is derived from assumptions based on past optimizations of injector parameters for HIF, and the desired pulse duration at the end of the 500 $\mathrm{MeV}$ accelerator. At injection, we have assumed a line charge density $\lambda_{\mathrm{o}}=0.2 \mu \mathrm{C} / \mathrm{m}$ at $2 \mathrm{MeV}$, $\mathrm{Rb}^{+}$ions. (Choosing $\mathrm{Cs}^{+}$, a heavier alkali ion would increase the kinetic energy somewhat, but would allow similar source technology. While it would have alleviated some of the constraints for an equal number of beams, we chose not to because it would have increased the kinetic energy somewhat, and it would not change the main conclusions.) At $500 \mathrm{MeV}$, for driver efficiency, it is desirable to have a pulse duration of a few-hundred ns at the end of the accelerator and the beginning of the drift compression section. Motivated by longitudinal phasespace constraints at the target for the main pulse, we have chosen a pulse duration of $\tau_{i}=5.33$ $\mu \mathrm{sec}$ at injection corresponding to an initial bunch length of 11.3 meters. This requires that voltage control (including a correction system) keep the beam energy within $\delta< \pm 0.2 \%$ of its nominal value, and this requirement is similar to that of previous drivers. For an injection kinetic energy of $\mathrm{T}=2 \mathrm{MeV}$ this corresponds to a longitudinal emittance of

$\varepsilon_{\| \mathrm{i}}=\frac{\tau_{\mathrm{i}}}{2} \mathrm{~T} \delta=\left(\frac{5.33 \mu \mathrm{s}}{2}\right)(2 \mathrm{MeV})(0.002)=0.011 \mathrm{eV} \cdot \mathrm{s}$

The half widths of the pulse duration and energy spread are used, and the implications for chromatic aberration are discussed in Sec. 12. It would not pay to try to improve the required voltage control to much better than $\pm 0.2 \%$ because other effects such as transverse-longitudinal coupling are also expected to produce a longitudinal emittance $\geq 0.011 \mathrm{eV} \cdot \mathrm{s}$.

The electrostatic quadrupoles transport a nearly constant $\lambda$ irrespective of energy so the bunches are accelerated at constant length through the accelerator. The resulting numbers of beams dedicated to each part of the power profile are shown in Table 1, along with some beam parameters at injection and at the end of acceleration. Based on the target pulse shape requirements there would be a total of 876 beams divided into four induction linacs; two 50$\mathrm{MeV}$ linacs and two 500-MeV linacs with respectively 268 and 184 beams each. These should be viewed as approximate numbers. There are certain numbers of beams that pack neatly into a linac transport array with quadrupole symmetry that fits into a circular induction core. Bundles of 268 and 184 beams pack neatly, so the line charge density per beam in Table 1 can be decreased slightly from the assumed $0.2 \mu \mathrm{C} / \mathrm{m}$ to fit the 904 beam total. In contrast, the number of beams in the RPD is 120, and in the National Ignition Facility is 192. 


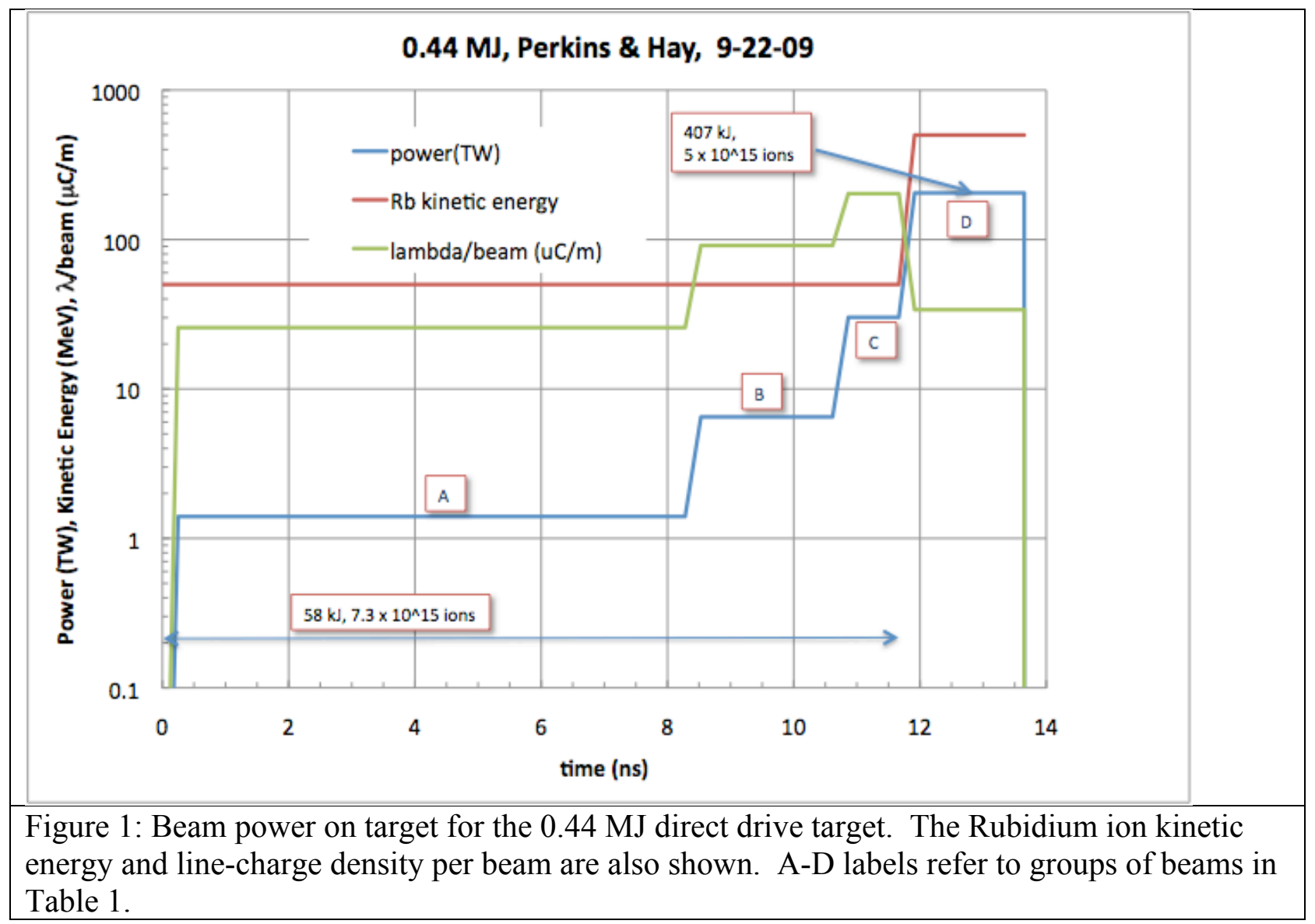

Within the accelerator the beam is space-charge dominated. It is made to be emittance dominated at the target (by plasma neutralization), where longitudinal and transverse phase space constraints due to focusing onto the target must both be satisfied. It is instructive to consider the combined six-dimensional phase space to get a sense of the relative difficulty for the wide range of kinetic energy required by the target.

The normalized transverse emittance is proportional to a beam's momentum, angular spread and radius:

$\varepsilon_{\mathrm{n}} \propto \mathrm{p}_{\mathrm{z}} \cdot \Delta \theta \cdot \mathrm{r}$

In the limit of emittance limited focal spots, the maximum allowable emittance is proportional to the convergence angle and focal spot radius. Thus, the allowable six-dimensional phase space volume is proportional to

$\mathrm{V}_{6 \mathrm{D}} \propto \frac{\tau}{2} \mathrm{~T} \delta \cdot\left(\mathrm{p}_{\mathrm{z}} \cdot \Delta \theta \cdot \mathrm{r}\right)^{2}$

and the number of ions per beam, $\mathrm{N}$, is proportional to power: 
$\mathrm{N} \propto \frac{\mathrm{P} \cdot \tau}{\mathrm{T} \cdot \mathrm{n}_{\mathrm{b}}}$

where $\mathrm{P}$ is the beam power, and $\mathrm{n}_{\mathrm{b}}$ is the number of beams.

Thus, the required phase space density is

$\rho_{6 \mathrm{D}} \equiv \frac{\mathrm{N}}{\mathrm{V}_{6 \mathrm{D}}} \propto \frac{\mathrm{P}}{\mathrm{n}_{\mathrm{b}} \mathrm{T}^{3} \delta \cdot \Delta \theta^{2} \cdot \mathrm{r}^{2}}$

Note the steep dependence on kinetic energy. As can be seen from this analysis and the subsequent sections, we find that several accelerator physics challenges can be mitigated with ions of higher kinetic energy.

\begin{tabular}{|c|c|c|c|}
\hline & foot pulse & $\begin{array}{c}\text { main pulse } \\
\text { (beam group } D \text { ) }\end{array}$ & units \\
\hline N_beams & 536 & 368 & \# \\
\hline N_beams (beam group A) & 106 & 0 & \# \\
\hline N_beams (beam group $B$ ) & 138 & 0 & \# \\
\hline N_beams (beam group C) & 292 & 0 & $\#$ \\
\hline $\mathbf{I}_{\mathbf{i n j}}$ & 0.426 & 0.426 & Amps \\
\hline $\mathbf{T}_{\text {inj }}$ & 2 & 2 & $\mathrm{MeV}$ \\
\hline$\lambda_{\text {inj }}$ & 0.2 & 0.2 & $\mu \mathrm{C} / \mathrm{m}$ \\
\hline $\mathbf{L}_{\text {beam, inj }}$ & 11.3 & 11.3 & meters \\
\hline$\tau_{\text {inj }}$ & 5.33 & 5.33 & $\mu \mathrm{sec}$ \\
\hline$\tau$ (end of acc) & 1.06 & 0.34 & $\mu \mathrm{sec}$ \\
\hline I(end of acc) & 2.14 & 6.78 & Amps \\
\hline Energy/beam (end of acc) & 0.114 & 1.14 & kJ \\
\hline$<E>$, acceleration gradient & 0.3 & 1.3 & $\mathrm{MV} / \mathrm{m}$ \\
\hline Accelerator length & 0.17 & 0.63 & $\mathrm{~km}$ \\
\hline \multicolumn{4}{|c|}{ drift compression, un-neutralized --> neutralized } \\
\hline L (drift compression) & & 230 & $\mathrm{~m}$ \\
\hline
\end{tabular}

\section{Fusion Chamber}

It is highly desirable to utilize thick liquid protection of the first structural wall for any HIF power plant design. Thus we assume some form of liquid jets (Li, Flibe or similar) oscillating within the target chamber, creating a pocket or void around the target with lines of sight between the jets to accommodate the ion beams. An internally consistent design was worked out for the RPD, but a corresponding effort has not been carried out for this study, which uses a smaller chamber (4 m cavity radius). Other approaches have been suggested recently, such as a rotating vortex of liquid at the periphery of the target chamber. We have not evaluated these options in 
detail, but rather confined the discussion here to more general observations regarding thick liquid protection and the direct drive target under consideration.

The most straightforward approach to direct drive illumination on the fusion target is to distribute a sufficient number of beams over $4 \pi$ steradians, but this is generally incompatible with flowing jets for radiation protection. In this study, we have simply assumed that it will be possible to modify the existing target design to accept aspherical illumination geometry without degrading target performance. Runge and Logan [Ru-09] have performed some preliminary calculations of targets that can use beams that are are in two bundles closer to the polar axis and directed preferentially toward the equatorial plane of the target. Thus there are two bundles of beams approaching the target (vs uniform spacing over $4 \pi$ ) yielding some space and solid angle for the injection of liquid jets.

Currently the calculations of Runge and Logan assume that the beam focal spots are hollow. The beam hollowing is assumed to be produced by rapidly rotating a small focal spot in a circular motion. In addition to the engineering issues of this approach, the required focal spot must be significantly smaller than the target size. This small focal spot size would make it even more difficult to design the accelerator.

From Sec. 3, there are 904 beams which must be arranged advantageously around the target chamber, the two bundles occupy $27 \%$ of the $4 \pi$ solid angle, including clearance between the beam edge and the liquid jets and required space between neighboring beams. The remainder is available for more liquid jets and tritium breeding. The occupancy and spacing of final focusing elements at the reactor chamber wall is addressed in Sec. 12.

\section{Driver System}

In previous years, the world's accelerator community was involved in examining the main accelerator types for fusion. These included: one synchrotron filling several storage rings from each of which several beams are kicked out simultaneously to obtain the power and energy desired; an r.f. linac filling several storage rings, as in the previous system; induction recirculators, which utilize several revolutions in one or more rings to enable multiple use of the beam transport and acceleration system; and induction linacs, which have the ability to amplify the current with acceleration. In induction accelerators, the current increases mainly from the increased speed of the ions, and additionally from the possibility of shortening of the bunch length. In this design, the physical bunch length is shortened only in the drift compression section. We have been pursuing the induction linac approach because these accelerators have routinely accelerated short, kilo-ampere (electron) beams and seem well matched to the desired repetition rates of a few pulses per second. In addition, because of the slow speed of the ions, it is possible to measure the beam and the acceleration voltage and feed forward information to correct the energy and current evolution of a single bunch.

The basic beam parameters at the target are shown in Table 2 . 


\begin{tabular}{|l|l|}
\hline Ion type & $\begin{array}{l}\mathrm{Rb}^{+} 84.91 \mathrm{amu}, \text { stripped to higher charge state } \\
\text { inside the fusion chamber }\end{array}$ \\
\hline Total energy & $0.44 \mathrm{MJ}$ \\
\hline Ion kinetic energies & $50 \mathrm{MeV}, 500 \mathrm{MeV}$ \\
\hline Power distribution & {$[$ See Sec. 3$]$} \\
\hline Final pulse duration & {$[$ See Sec. 3$]$} \\
\hline Spot radius & $0.5 \mathrm{~mm}$ \\
\hline Number of beams & 368 at $500 \mathrm{MeV}$ \\
& 536 at $50 \mathrm{MeV}$ \\
\hline & \\
\hline
\end{tabular}

Table 2: Basic beam parameters at the target

In order to maximize driver efficiency and minimize driver cost, it is usually desirable to accelerate as many beams as possible in as few individual accelerators as possible. On the other hand, the use of multiple accelerators simplifies the beam transport system between the accelerator and the fusion chamber. Since our example driver requires significantly more beams than previous multi-GeV examples, we have balanced these competing considerations by assuming four induction linacs, i.e., two each at 50 and $500 \mathrm{MeV}$, which are positioned on opposite sides of the fusion chamber as shown in Fig. 2. These provide the power blocks of beams that are directed onto the target as prescribed for a high gain implosion.

Beams from each linac are separated into groups of $\approx 16$ each at the end of acceleration, with each group containing only beams of a particular power block. The total path length to the target and the velocity ramp to provide the final block duration is then identical within a beam group, and there are only very small differences among groups. The beams are compressed longitudinally from an energy ramp (10-15\%) imposed near the exit of the accelerator.

The path length from the linacs to the target is about 230 meters for all beams, with small differences among blocks due to their differing final arrival times. However, these drift paths are curved, typically $\approx 135^{\circ}$ total bending, prior to the final focus magnets. The path length requirements are severe, so the bends must be adjusted for different beams so that all beams within a group arrive at the target simultaneously. The path layout of magnetic bends and quadrupoles must minimize the chromatic aberration of focal spot size and position caused by the $\pm 2.5-3.5 \%$ velocity ramp and the instantaneous velocity spread due to the longitudinal emittance of the beam. Compression from an initial $11.3 \mathrm{~m}$ at the end of acceleration to a few $\mathrm{cm}$ at the target takes place continuously over the entire $230 \mathrm{~m}$ distance. The beams are neutralized by plasma background electrons during the final $30-60 \mathrm{~m}$ of transport in order to eliminate the large repulsive space charge force in both the transverse and longitudinal directions.

The final focus magnets are high field solenoids $\left(\approx 10 \mathrm{~T}\right.$ for $\left.500 \mathrm{MeV} \mathrm{Rb}{ }^{+}\right)$since magnetic quadrupoles are predicted to disrupt charge and current neutrality provided by the injected plasma. Also, solenoids usually have smaller chromatic aberrations from random and ramped momentum variations than uncorrected quadrupoles lattices. 
Just prior to plasma neutralization a pulsed electric quadrupole system puts a small envelope oscillation onto the compressing beams. The purpose of this operation is to compensate for the time depended shift of the focal length of the solenoids associated with the velocity ramp.

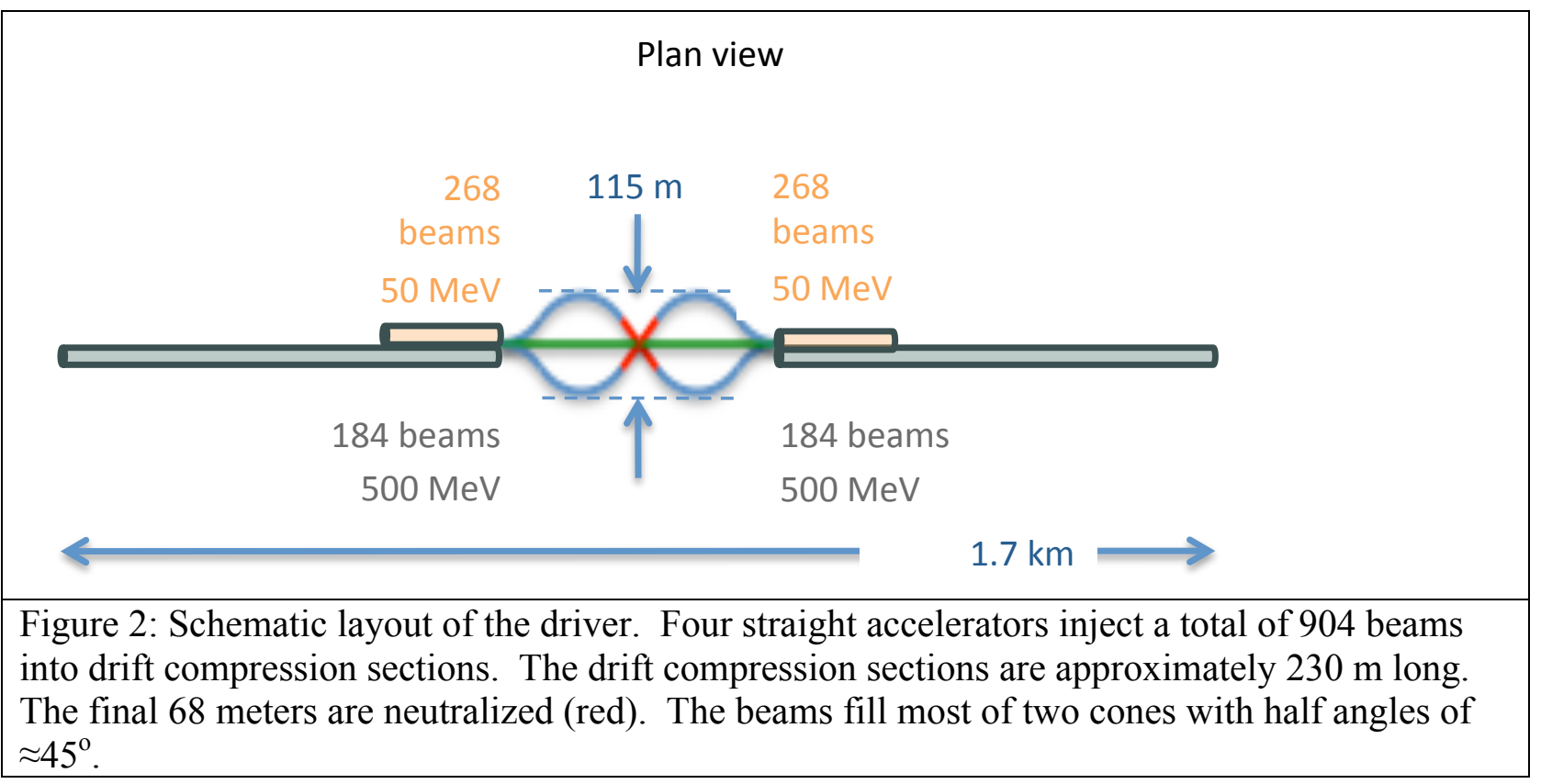

The distance from the fusion target to a final focus solenoid is about $5 \mathrm{~m}$, with the last $\approx 1 \mathrm{~m}$ occupied by shielding against fusion neutrons. A complex set of FLIBE jets covers $\approx 90 \%$ of the total solid angle looking out from the target. Stripping of the $\mathrm{Rb}^{+}$to higher charge state by chamber vapor must be avoided until the beams are clear of the solenoid fringe field. Beam passage through the vapor leads to stripping and multiple scattering, which further erode the focal spot intensity.

The induction linacs begin with large integrated sets of ion sources of a triode design operating with extraction voltage $60-80 \mathrm{kV}$ and producing 0.426 Amps each. This is based on past systems studies and also the $2 \mathrm{MV}$ injector design of the existing HCX. [Bi-05, Pr-05]. The ion energy is raised to $2 \mathrm{MeV}$ in a high gradient injector column and matching section that also compresses the beams transversely from $\approx 5 \mathrm{~cm}$ to $1.2 \mathrm{~cm}$ radius and bends them into the linac focal array. The initial pulse duration is $5.33 \mu \mathrm{s}$.

In the induction linacs, we explore the concept of multiple parallel beams transported by arrays of electrostatic quadrupoles. The benefit of this choice is still unclear. Electrostatic focusing may lead to a shorter development path compared to superconducting quadrupole arrays, but it implies the use of electrostatic quadrupoles well above the energy beyond which superconducting arrays are capable of transporting higher charge-density beams. Thus, the shorter development path for electrostatic focusing is offset by a higher-cost accelerator structure (more flux or volt-seconds) because the transverse dimension of the array may be greater, and 
the bunch duration is longer (requirement to accelerate at nearly constant bunch length). The cost versus benefit is not yet settled.

Beams emerging from the $50 \mathrm{MeV}$ linacs have much lower velocity than the $500 \mathrm{MeV}$ beams (by $\approx 10^{1 / 2}$ ), so they must be accelerated $\sim 15 \mu$ s earlier so that they arrive at the target on schedule. Except for their different diameters and core sizes to accommodate the different numbers of beams, the 50 and $500 \mathrm{MeV}$ linacs are similar until the $50 \mathrm{MeV}$ point. The velocity ramps of $\pm 3.5 \%$ are imposed near the end of acceleration to beams in blocks A-C, and $\pm 2.5 \%$ to block D. Small adjustments in the velocity ramps as well as path-length differences are applied separately to the beams of blocks A, B and C in order to effect the desired arrival time and duration on target of each block.

Details of the driver system are described in subsequent sections.

\section{Beam Source and Injector}

The beam source and injector geometry is a modest scale-up from $\mathrm{HCX}\left(\mathrm{K}^{+}, 1 \mathrm{MeV}, 0.18 \mathrm{~A}\right.$ with $\mathrm{a}_{\mathrm{s}}=0.05 \mathrm{~m}$ radius emitting surface at $1050-1100^{\circ} \mathrm{C}$ ). For the $\mathrm{Rb}^{+}$driver we again use source radius $\mathrm{a}_{\mathrm{s}}=0.05 \mathrm{~m}$, effective extraction voltage of $80.7 \mathrm{kV}$ and extraction gap $\mathrm{d}=\mathrm{a}_{\mathrm{s}}$. Application of the Child-Langmuir law for space-charge limited flow gives

$\mathrm{J}_{\mathrm{s}}=5.46 \times 10^{-8} \sqrt{\frac{\mathrm{V}_{\mathrm{ext}}^{3}}{\mathrm{Ad}^{4}}}=54.3 \mathrm{~A} / \mathrm{m}^{2}$

$\mathrm{I}=\pi \mathrm{a}_{\mathrm{s}}^{2} \mathrm{~J}_{\mathrm{s}}=0.426 \mathrm{~A}$

The transverse emittance associated with the hot emitting surface is:

$\varepsilon_{\mathrm{n}}=6.55 \times 10^{-5} \sqrt{\frac{\mathrm{T}_{\mathrm{s}}(\mathrm{eV})}{\mathrm{A}}} \mathrm{a}_{\mathrm{s}}=1.22 \times 10^{-7} \mathrm{~m}$

This is the intrinsic normalized edge emittance with $\mathrm{Ts}=1100^{\circ} \mathrm{C} \rightarrow 0.118 \mathrm{eV}$. Extracted charged particle beams almost always exhibit a larger emittance than the intrinsic emittance at the source surface. In HCX, for example, the intrinsic emittance for the $1-\mathrm{MeV} \mathrm{K}^{+}$beam is 1.66 $\mathrm{x} 10^{-7} \mathrm{~m}$, while the measured emittance after matching was $\approx 4.5 \times 10^{-7} \mathrm{~m}$, or almost a factor three greater than at the emitting surface [Pr-05]. Thus, we adopt an emittance after matching of $3.75 \times 10^{-7} \mathrm{~m}$. Other methods of scaling the HCX results to these parameters yield higher emittance, so there is some uncertainty to this adopted emittance.

$\mathrm{The} \mathrm{Rb}^{+}$ions are further accelerated through the $2 \mathrm{MV}$ injector in a multi-beam high gradient structure; this reduces the line-charge density to $\lambda=0.2 \mu \mathrm{C} / \mathrm{m}$ as desired for transport in the linac by electric quadrupoles. Compression of the beam radius to the average value of about $1.2 \mathrm{~cm}$ is achieved with a special set of high voltage electrodes with irregular spacing and diameters (as in HCX). Several outstanding issues for injector development are apparent: the implied funneling of curved beam paths into the linac, heat management of the large source array and longevity of the $\mathrm{Rb}^{+}$sources at $5 \mathrm{~Hz}$ operation. The funneling of lower current and lower energy beams was successfully demonstrated in a beam merging experiment [Se-00].

\section{$7 \quad$ Induction Linac with Electrostatic Focusing}

Transportable line change density $\lambda$ is roughly proportional to the quadrupole electrode potential when other parameters such as longitudinal electrode occupancy $\eta$ are optimized. To prevent 
electrical breakdown on high-voltage feed-throughs the electrode potential is presently limited to $\phi \leq \pm 80 \mathrm{kV}$, which is further reduced for long-term reliability. The feed-throughs can certainly be improved and eventually the higher limit would be metal-to-metal breakdown of the quadrupole electrodes. This consideration results in $\lambda \leq .25 \mu \mathrm{C} / \mathrm{m}$ throughout the linac. A constant $\lambda$ and therefore constant pulse length $\ell$ during acceleration is referred to as a "load and fire" scenario. Following the $V_{0}=2.0 \mathrm{MV}$ injector, beam pulses are introduced into the linac focal arrays without immediate acceleration. When the beams are fully loaded, the induction modules are powered (gently at first to avoid excessive longitudinal emittance growth) such that the entire pulse feels essentially the same accelerating gradient at any particular time. The mean gradient $\mathrm{E}$ is regarded as either a function of time or of cumulative voltage $V(t)=m v^{2} / 2 e ; \mathrm{E}=\mathrm{E}(V)$. Thus at any location $z$ the cumulative voltage increases from the pulse head to the pulse tail. This causes the "natural" velocity tilt associated with load and fire and it causes the beam radii to decrease slightly from head to tail. These are concerns mainly at the very front of the accelerator, and become minor as acceleration progresses.

Due to the large number of parallel beams (see Fig. 3) we select focal array parameters that minimize its transverse size while accommodating the mean $\lambda=.2 \mu \mathrm{C} / \mathrm{m}$. This value is actually $8 \%$ above that required for the target but some losses from e.g. stripping and halo scrape-off are expected downstream. Minor variations of $\lambda$ above and below its mean are expected from the finite acceleration gaps, details of the accelerating waveform, and rise/fall of the pulse ends.

Injection parameters (at $\left.V_{0}\right)$ are:

$\begin{array}{ll}\text { pulse duration } & \tau_{o}=5.33 \mu \mathrm{s} \\ \text { velocity } / \mathrm{c} & \beta_{0}=.00711 \\ \text { pulse length } & \ell_{0}=11.35 \mathrm{~m} \\ \text { line change density } & \lambda_{0}=.200 \mu \mathrm{C} / \mathrm{m} \\ \text { undepressed tune } & \sigma_{u}=80^{\circ} \\ \text { lattice half period } & L_{0}=.26 \mathrm{~m} \\ \text { quadrupole occupancy } & \eta_{0}=.70 \\ \text { electrode potential } & \phi= \pm 67 \mathrm{kV} \\ \text { channel bore } & b=.03 \mathrm{~m} \\ \text { maximum beam edge radius } & a_{\max }=.016 \mathrm{~m} \\ \text { mean beam edge radius } & \bar{a}=.012 \mathrm{~m}\end{array}$

For initial emittance $\varepsilon=\varepsilon_{\mathrm{n}} / \beta_{\mathrm{o}}=5.27 \times 10^{-5} \mathrm{~m}$ the depressed tune $\sigma=2 \mathrm{~L} \varepsilon / \overline{\mathrm{a}}^{-2}=10.9^{\circ}$ is negligible, so the beam radii are determined from the space charge limit formula:

$$
\frac{\lambda}{\pi \varepsilon_{0} \mathrm{~V}} \frac{\mathrm{L}^{2}}{\mathrm{a}} \approx 2\left(1-\cos \left(\sigma_{\mu}\right)\right)
$$




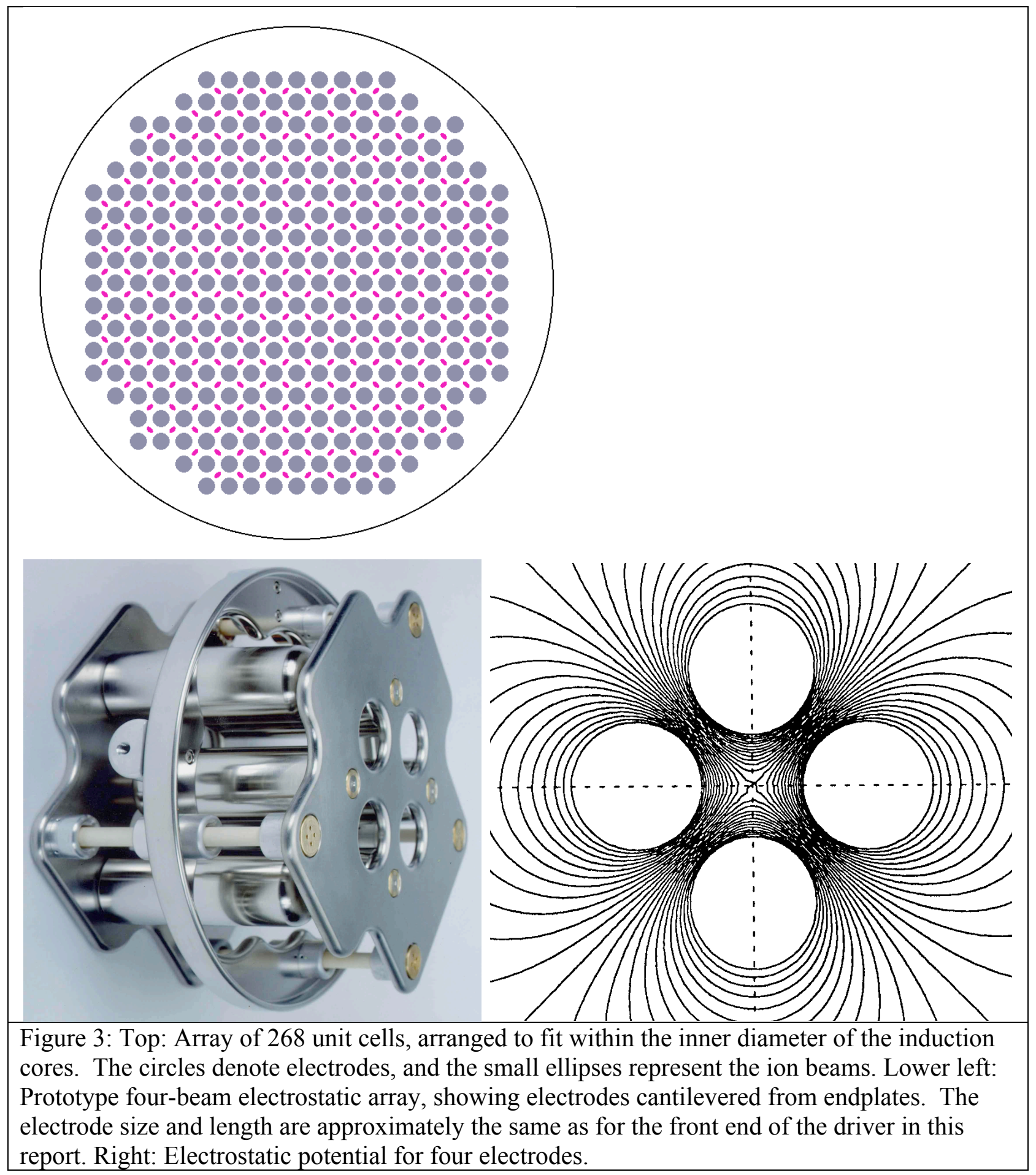

The electrode radii are $(8 / 7) b$, a value that zeros the dodecapole aberration and largely shields beams from each other. In this configuration the lattice pitch (diagonal of a unit cell) is $\mathrm{S}=\sqrt{2} \mathrm{p}=\sqrt{2}(1+8 / 7) \mathrm{b}=.0909 \mathrm{~m}$. A roughly circular array has diameter 
$\mathrm{D}(268$ beams $) \approx 20 \mathrm{p}=1.82 \mathrm{~m}$

$\mathrm{D}(184$ beams $) \approx 17 \mathrm{p}=1.64 \mathrm{~m}$

An additional $\sim .2 m$ of array diameter is required for high voltage ceramic insulators, vacuum interface and associated clearance interior to the induction cores.

The initial lattice half period $L_{0}=.26 \mathrm{~m}$ accommodates an acceleration gap $(\sim .03 \mathrm{~m})$, a pair of high voltage plates $(\sim .015 \mathrm{~m}$ each), staggered electrodes attached to the plates $(\sim .17 \mathrm{~m})$, and electrode-to-plate gaps $(\sim .03 \mathrm{~m})$. The beams pass through holes in the plates and are surrounded by electrodes except in the accelerating gaps. The effective occupancy $\eta$ is a little larger than the electrode length divided by $L$, due to the \pm electrodes incomplete longitudinal overlap. This feature also introduces a very small sextupole aberration.

\section{Linac Scaling and Induction Core Requirements}

As mentioned, line charge density $\lambda$ and pulse length $\ell$ are constant during acceleration. A rough scaling law for the lattice half period $L$ follows if we also hold $\eta, \sigma_{u}$, and $\bar{a}$ constant:

$$
\mathrm{L}=\mathrm{L}_{0} \sqrt{\frac{\mathrm{V}}{\mathrm{V}_{0}}}
$$

As $L$ increases it is necessary to construct the focal arrays by combining short sections similar to the array at $V_{0}$ to avoid the use of long cantilevered electrodes and to divide the accelerating voltage into smaller increments.

The induction cores/pulsers provide a useable constant volt·second product per meter:

$$
\mathrm{E} \tau=\mathrm{E}_{0} \tau_{0}=0.5 \mathrm{~V} \cdot \mathrm{s} / \mathrm{m}
$$

Since $\tau=\tau_{0} \sqrt{V_{0} / V}$ we have

$$
\mathrm{E}(V)=\frac{\mathrm{E}_{0} \tau_{0}}{\tau}=\mathrm{E}_{0} \sqrt{\frac{V}{V_{0}}}
$$

The initial gradient is $\mathrm{E}_{0}=93.8 \mathrm{kV} / \mathrm{m}$. At the linac ends (50 and $\left.500 \mathrm{MeV}\right)$ the above scaling gives:

$$
\begin{aligned}
L_{50} & =1.3 \mathrm{~m} \\
\tau_{50} & =1.07 \mu \mathrm{s} \\
\mathrm{E}_{50} & =470 \mathrm{kV} / \mathrm{m}
\end{aligned}
$$




$$
\begin{aligned}
L_{500} & =4.11 \mathrm{~m} \\
\tau_{500} & =.337 \mu \mathrm{s} \\
\mathrm{E}_{500} & =1.48 \mathrm{MV} / \mathrm{m}
\end{aligned}
$$

The linac lengths are:

$$
\begin{aligned}
& (\text { to } 50 \mathrm{MeV})=\int_{V_{0}}^{50} \frac{d V}{\mathrm{E}(V)}=171 \mathrm{~m} \\
& (\text { to } 500 \mathrm{MeV})=\int_{\mathrm{V}_{0}}^{500} \frac{\mathrm{dV}}{\mathrm{E}(\mathrm{V})}=632 \mathrm{~m}
\end{aligned}
$$

We note that the constant occupancy of $\eta=0.7$ leaves $(1-\eta) \cdot L_{500}=1.23 \mathrm{~m}$ available axially to impart the $\mathrm{E}_{500} \bullet \mathrm{L}_{500}=6.1 \mathrm{MV}$ of acceleration in a lattice half-period. Since voltage hold-off of the full $6 \mathrm{MV}$ is prohibitive in a single gap (plus supporting plates), the acceleration must be distributed among a number of gaps. For the voltage holding criteria to be valid, the gaps must be isolated (e.g., interspersed with short quadrupoles of like-polarity focusing). Voltage hold-off is linear for short gaps, and has a $\mathrm{g}^{1 / 2}$ dependence for gap length $\mathrm{g}>1 \mathrm{~cm}$ [Fa-90]. For example, a design criterion of $(200 \mathrm{kV}) /(3 \mathrm{~cm}$ gap) would require 30 gaps, with a integrated acceleration gap length of $\approx 90 \mathrm{~cm}$. Since 30 quadrupoles are also needed, this implies unreasonably short quadrupoles. Therefore, $1.48 \mathrm{MV} / \mathrm{m}$ appears to be very difficult for this type of accelerator, and a lower gradient (longer linac) might be necessary. Further study is required.

The total useable core flux swings required for acceleration are

$$
\begin{aligned}
& (\text { to } 50 \mathrm{MeV})=85.3 \text { Volt }-\mathrm{sec} . \\
& (\text { to } 500 \mathrm{MeV})=316 \text { Volt }-\mathrm{sec} .
\end{aligned}
$$

Some additional core must be included for "ear fields" that hold beams to the desired length, with nearly constant $\lambda$.

The natural velocity tilt is approximately estimated using D. Judd's formula:

$$
\frac{\Delta v}{v}=\frac{v \text { tail }-v \text { head }}{\bar{v}}=\frac{\mathrm{E} \ell}{V}\left(\frac{1}{2}-\frac{d \log (\ell)}{d \log (V)}\right) .
$$

Here $\ell$ is constant so we have

$$
\frac{\Delta v}{v}=\frac{\mathrm{E} \ell}{2 V}=\frac{\mathrm{E}_{0} \ell_{0}}{2 \sqrt{V_{0} V}} .
$$

At 2, 50, and 500MeV this gives 


$$
\begin{aligned}
& \left(\frac{\Delta \mathrm{v}}{\mathrm{v}}\right)_{0}=.265 \\
& \left(\frac{\Delta \mathrm{v}}{\mathrm{v}}\right)_{50}=.0530 \\
& \left(\frac{\Delta \mathrm{v}}{\mathrm{v}}\right)_{500}=.0168
\end{aligned}
$$

The natural tilts at $50 \mathrm{MeV}$ and $500 \mathrm{MeV}$ are somewhat below the nominal values of 0.07 and 0.05 adopted for drift compression after acceleration. Therefore, the $500 \mathrm{MeV}$ linac must have programmed waveforms over the final $\sim 50 \mathrm{~m}$ of acceleration to raise the tilts to the required levels.

\section{Beam Separation at the End of Acceleration}

Up to the final energies of 50 and $500 \mathrm{MeV}$ the beams are focused with compact arrays of electrostatic quadrupoles. At the exits of the accelerators the beams are separated into smaller bundles of the same dense array and transported to various locations around the chamber. The beams are transported in bundles instead of individually because the bundles are more compact and much more economical than single beams.

Moving a quadrupole channel by smooth displacements results in the beam following the channel, with small betatron oscillations around the center of the channel with little damage to the beam quality. No dipoles as such are required to bend the beam. Inclusion of dipoles into the quad array is very difficult, because to bend all of the beams in one direction requires the electric field of all channels to be in that direction, with a continuous increase of voltage across the array. The quadrupole array is made of two intertwined equipotential surfaces. There is little space axially to put in dipoles, and here too the electric field must reverse after every beam channel or the potential builds up.

One solution to bending the beams is to use displaced or off-centered quadrupoles. Because the quad electric fields increase linearly from the center of the structure, $E_{x, y}=E^{\prime} \cdot x\left(\right.$ or $\left.E^{\prime} \cdot y\right)$ any point within the quad field can be thought of as the center of a dipole, with field $E d=\mathrm{E}^{\prime} \delta$, where $\delta$ is the distance from the center, plus a quadrupole field of the same gradient as before. Consider an $\mathrm{F}$ (focusing) quad in the $\mathrm{x}$-direction: if the beam were initially centered at $x=0$, moving it to negative $\mathrm{x}$ will put it into a dipole field which bends it toward the positive direction. Alternatively, moving the channel toward positive $\mathrm{x}$ has the same effect. However, if the quad were a $\mathrm{D}$ (defocusing) quad in $\mathrm{x}$, then a bend to the right or positive $\mathrm{x}$ would be accomplished by moving the channel towards negative $\mathrm{x}$.

Thus, one option for increasing the strength of the bend is to move all the F lenses in one direction and the D lenses in the opposite direction. In an array the F's and D's alternate in the compact arrays used for HIF, so the effect of moving the F's and D's in opposite directions is to distort the electrode shape somewhat. A typical distortion would be about $1.3 \mathrm{~mm}$ on a $26-\mathrm{mm}$ radius electrode. A much larger channel displacement, $\sim \mathrm{mm}$ is shown in Fig 4 to illustrate the alternating nature of the required electrode distortions. The electrode surfaces nearest the beam are made to follow the hyperbolic surface approximation and the distortions are relatively far 
from the beams. From such an array the bend angle increases linearly with distance. All beams within a quadrant are bent by equal amounts, away from the other quadrants. Only the electrodes along the axes need to be continually stretched until like in mitosis, they can be separated or split into two, and the array becomes four independent quadrants.

$$
\text { For } \begin{aligned}
\phi_{\mathrm{q}} & = \pm 75 \mathrm{kV} \\
\mathrm{E}^{\prime} & =\frac{2 \phi_{\mathrm{q}}}{\mathrm{b}} \cong \frac{150 \mathrm{kV}}{23 \mathrm{~mm}}=65 \mathrm{kV} / \mathrm{cm}
\end{aligned}
$$

and $\mathrm{E}=\mathrm{E}^{\prime} \delta=6.5 \mathrm{kV} / \mathrm{cm}$ is the bend field for a $1 \mathrm{~mm}$ displacement.

The resultant angle is

$$
\theta=\frac{\mathrm{E} \ell}{2 \mathrm{~T}} \approx \frac{(6.5 \mathrm{kV} / \mathrm{cm})(50 \mathrm{~cm})}{(2)(500 \mathrm{MV})}=3.3 \times 10^{-4} \mathrm{radian}
$$

The balance between aperture for the bend, bend aberration, and bend angle would require a small study for an optimum, but it is clear that the bend and transport would be over a substantial distance (10-20 meters), compatible with the first portion of drift compression anyways.

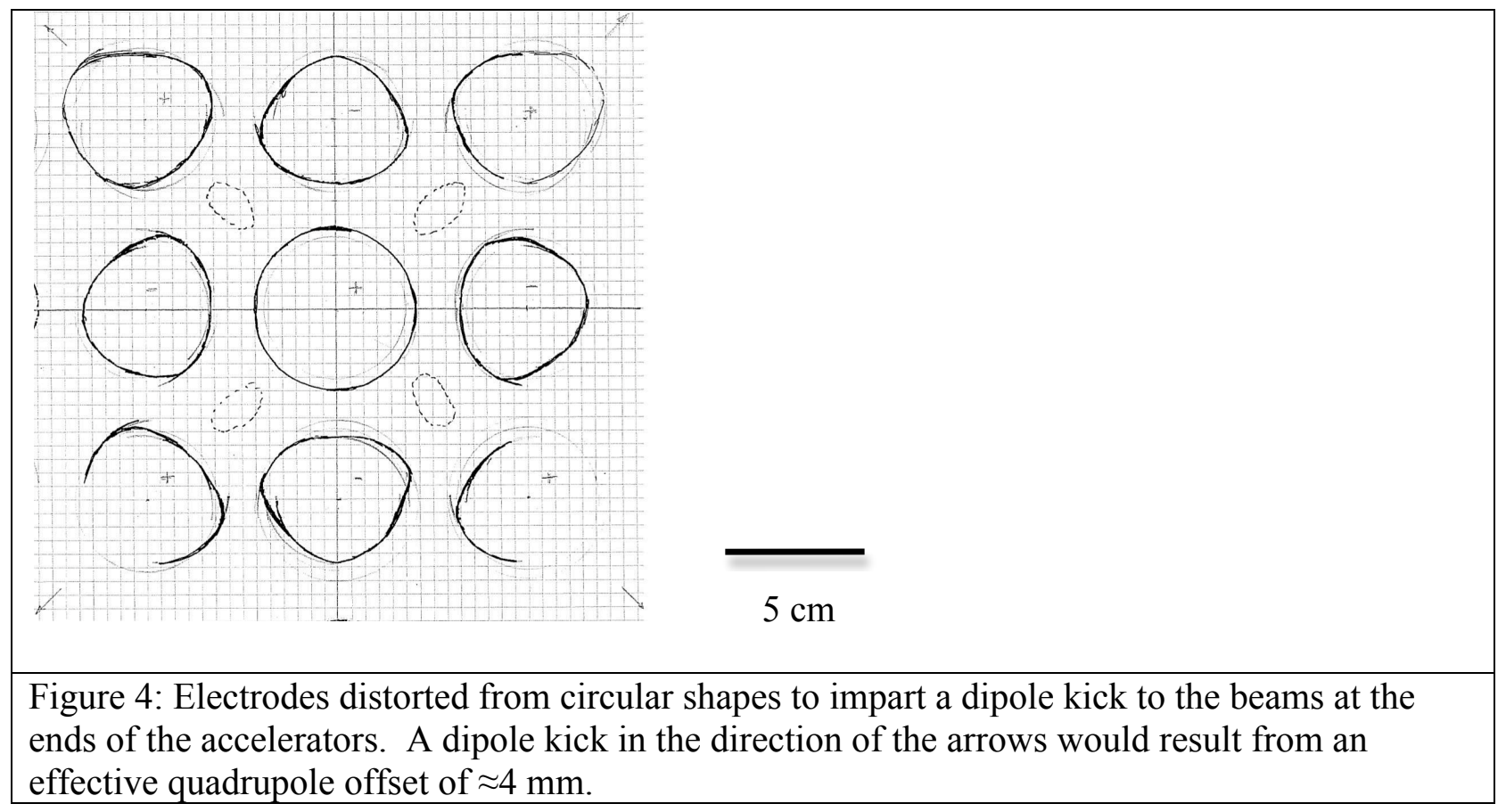

\section{Velocity Ramps and Longitudinal Compression}

Several factors limit longitudinal beam compression. The most fundamental are space-charge effects and longitudinal emittance. Consider first the space-charge effects. 
A total drift compression distance of $230 \mathrm{~m}$ matches the initial pulse length $\left(\ell_{0}=11.3 \mathrm{~m}\right)$ and approximate velocity tilt $\Delta \mathrm{v} / \mathrm{v}=0.05 \approx 11.3 / 230$ (or $\pm 2.5 \%$ about the ion velocity at $500 \mathrm{MeV}$ ). This distance starts at the end of the linacs and ends at the target, so the complications of beam separation, bending, envelope compensation, neutralization, and final focus all occur along the drift lines. A shorter drift distance requires increased tilt, which increases dispersion in the bends, makes the bend layout difficult, and increases the required envelope compensation for final focus.

It is necessary to neutralize the beams prior to final focus to prevent the stalling of compression by the longitudinal space charge force. This feature is much more pronounced for $50 \mathrm{MeV}$ than $500 \mathrm{MeV}$ and leads for $50 \mathrm{MeV}$ to an increase of tilt to $\Delta \mathrm{v} / \mathrm{v}=0.07$ (or $\pm 3.5 \%$ ).

Most of the tilt is applied during the final stage of acceleration in the linacs. But to fine tune the arrival times and durations of the three $50 \mathrm{MeV}$ beam groups some minor adjustments are made just after the separation system while the beams are still clustered in group subsets. To accommodate the bending geometry it appears that a final separation of all beams in each subset can be delayed until about $100 \mathrm{~m}$ from the target.

The approach is to neutralize before longitudinal space charge plays a large role in the evolution of the bunch length. To model this process an approximate dynamical equation for the beam length $\ell(z)$ is adopted:

$$
\begin{aligned}
& \frac{\mathrm{d}^{2} \ell}{\mathrm{dz}}=\frac{\mathrm{C}(\mathrm{z})}{\ell^{2}} \\
& C(z)=\left\{\begin{array}{cc}
\frac{6 \lambda_{0} \ell_{0} \mathrm{~g}}{4 \pi \varepsilon_{0} V} & \text { in vacuum } \\
0 & \text { neutralized }
\end{array}\right.
\end{aligned}
$$

Using $(\mathrm{b} / \overline{\mathrm{a}})=1.5$, then $\mathrm{g}=2 \cdot \log (\mathrm{b} / \overline{\mathrm{a}}) \approx 0.811, \ell(0)=\ell_{0}=11.3 \mathrm{~m},(\mathrm{~d} \ell / \mathrm{dz})_{0}=-(\Delta \mathrm{v} / \mathrm{v})_{0}$. Integration of this equation suggests a good location to turn off $C$, i.e. neutralize, in order to focus at $230 \mathrm{~m}$.

For beams at $500 \mathrm{MeV}, \mathrm{C}=1.98 \times 10^{-4}$.

Neutralization at $205 \mathrm{~m}$ yields a longitudinal focus at $230 \mathrm{~m}$. Without neutralization compression stalls at $231.6 \mathrm{~m}$ with minimum beam length $\approx 0.155 \mathrm{~m}$ (too long).

For beams at $50 \mathrm{MeV}, \mathrm{C}=1.98 \times 10^{-3}$.

A problem appears: to focus at $230 \mathrm{~m}$ requires neutralization at $50 \mathrm{~m}$. This is not allowed because subsequent bending must be done in vacuum. One reasonable modification of the system is to increase tilt to 0.07 in the $50-\mathrm{MeV}$ linacs. Then neutralization is applied at $168 \mathrm{~m}$ to get a focus at $230 \mathrm{~m}$. The final $230-168=62 \mathrm{~m}$ has a straight axis and neutralized transport in 
solenoids up to the fusion chamber would be employed. The space charge force reduces the tilt to 0.012 just prior to neutralization; this is a benefit for final focus dynamics, but there is a great sensitivity to the location of the onset of neutralization.

Now consider the effects of longitudinal emittance. The worst case occurs in the $50 \mathrm{MeV}$ foot pulse. The last part of the foot pulse, according to Figure 1, has a duration of $1 \mathrm{~ns}$. Therefore the resulting energy spread at the target is at least $0.011 \mathrm{eV} \cdot \mathrm{s} / 0.5 \mathrm{~ns}= \pm 22 \mathrm{MeV}$ or $\pm 44 \%$. Even if the target could accept this large energy spread, we have been unable to find a method of focusing such a beam to the required focal spot size. In the middle of the final focus solenoid ( $7.5 \mathrm{~m}$ from the target) the pulse duration is $\approx 9 \mathrm{~ns}$ and energy spread $\approx 5 \%$, still much too large to focus onto the target. Another very acute difficulty is that the ion pulse duration set by Fig. 1 cannot be realized, because the velocity tilt is smaller than the implied velocity spread. Improvements in target design will be necessary to cope with this difficulty. We did not rework the driver design since it does not appear to change the general conclusions in this paper.

\section{Achromatic Bends}

A bend system is needed to bring the beams to the fusion chamber at their specified ports and pointed at the target. Transport over the final 25 or $62 \mathrm{~m}$ is a neutralized straight shot and free of bends and quadrupoles; solenoids are used for transport and final focus there. Consider the extreme case of a beam brought to the chamber at an angle of $45^{\circ}$ from the linac axis. Roughly this could be done using an initial $45^{\circ}$ bend with mean radius of curvature $\langle\rho\rangle=90.90 \mathrm{~m}$, followed by two reverse $45^{\circ}$ bends with radii of $90.90 \mathrm{~m}$ and $32.11 \mathrm{~m}$, and finally a $62.0 \mathrm{~m}$ straight path to the target. This beamline reaches a maximum of 53.3-m displacement from the linac axis. Other beamlines that enter the chamber at less than $45^{\circ}$ actually require a similar amount of bending on a more complicated path to make equal total path lengths from the linac. An additional complication is the need to interleave the 50 and $500 \mathrm{MeV}$ beam groups from adjacent linacs. These latter topics would be treated in a more detailed design than the present exercise.

One important feature that constrains the design of the drift compression section is the orbit dispersion by the bends acting on the large velocity tilt. For a particular beam segment with velocity $v+\delta v$ the dispersion $X(z)$, which is the off-axis displacement, roughly satisfies the dynamic equation

$$
\frac{\mathrm{d}^{2} \mathrm{X}}{\mathrm{dz}^{2}}=-\mathrm{k}_{\beta}^{2} \mathrm{X}+\frac{\delta \mathrm{v}}{\mathrm{v}} \frac{1}{\langle\rho\rangle}
$$

Here $\mathrm{k}_{\beta}=\sigma_{0} / 2 \mathrm{~L}$ is the mean betatron frequency from the transport quadrupoles. The mean value of $X$ is:

$$
\langle X\rangle=\frac{\delta v}{v} \frac{1}{\langle\rho\rangle} \frac{1}{k_{\beta}^{2}}
$$


If quadrupoles and bends are arranged ideally then $X(z)$ may have only moderate oscillations around $\langle X\rangle$ and return to axis at the end of the bend system. This is the condition of first order achromaticity and is essential for bringing the beam onto the target as required and without jitter.

Large $\langle X\rangle$, say greater than $\pm 0.0 .1 \mathrm{~m}$, adversely impacts quadrupole and bend design. It also exposes the beam to higher order aberrations that increase its occupied phase space volume. To make $\langle X\rangle$ small we must make $\langle\rho\rangle$ or $k_{\beta}$ large. Since $\langle\rho\rangle$ is essentially determined by system geometry we consider increased $k_{\beta}$, which requires decreased $L$.

$$
\begin{aligned}
\text { Example } \begin{aligned}
\mathrm{L} & =4.11 \mathrm{~m} \\
\sigma_{0} & =80^{\circ} \\
\langle\rho\rangle & =90.90 \mathrm{~m} \\
\delta \mathrm{v} / \mathrm{v}= \pm \frac{\text { tilt }}{2}= \pm .025 & \text { (final linac value) }
\end{aligned} \\
\mathrm{k}_{\beta}=\frac{\sigma_{0}}{2 \mathrm{~L}}=\frac{80 \pi / 180}{2 \times 4.11}=0.170 \mathrm{~m}^{-1} \\
\langle\mathrm{X}\rangle=\frac{( \pm .025)}{90.90 \times(.170)^{2}}= \pm 0.00952 \mathrm{~m}
\end{aligned}
$$

If we reduce $L$ to $2.0 \mathrm{~m}\langle X\rangle$ is reduced to the moderate value of \pm 0.00225 .

Transport in the bend system is carried out using superconducting quadrupoles with pole field in the range 0.3-3.0T depend on local $L, \lambda$, and $V$. Multiple channel arrays of quadrupoles for sets of beams would be used to minimize the cryostat expense. Conceptual designs for superconducting quadrupole arrays have consisted of alternating focusing and defocusing channels transversely, where the external field of any quadrupole becomes the internal field of its neighbor. The array is surrounded by edge windings to maintain field quality in the apertures at the periphery of the array. The array also has an axial overhang to make the fringe field of every channel identical.

The mean bend field is quite low. For $500 \mathrm{MeV} R b^{+}$the magnetic rigidity is, $(\mathrm{B} \rho)=M v / e=29.7$ $\mathrm{T} \cdot \mathrm{m}$, so to produce $\langle\rho\rangle=90.90 \mathrm{~m}$ we need only

$$
\langle\mathrm{B}\rangle=\frac{\langle\mathrm{B} \rho\rangle}{\langle\rho\rangle}=\frac{29.7}{90.90}=0.327 \mathrm{~T} .
$$

Dipole bend magnets with longitudinal occupancy $\eta_{B}=0.33$ would have

$$
\mathrm{B}=\langle\mathrm{B}\rangle / \eta_{\mathrm{B}}=0.98 \mathrm{~T}
$$


and alternate with quadrupoles.

\section{Neutralization and Final Focus}

The $R b^{+}$beams are focused by strong solenoids positioned just outside the fusion chamber, with the solenoid ends $5.0 \mathrm{~m}$ from the fusion target. The chamber has an inner radius of $4.0 \mathrm{~m}$ surrounded by $1 \mathrm{~m}$ of protective shielding. This is penetrated by 904 cylindrical beam ports of $\sim 7$ $\mathrm{cm}$ radius each. Every final focus solenoid occupies $3.6 \times 10^{-3}$ steradians, equivalent to a $30 \mathrm{~cm} \mathrm{x}$ $30 \mathrm{~cm}$ angular patch $5.0 \mathrm{~m}$ from the target. The array of ports covers two $90^{\circ}$ cones on opposite sides of the chamber, with roughly $27 \%$ total occupancy of the full $4 \pi$ solid angle. The $500 \mathrm{MeV}$ and $50 \mathrm{MeV}$ ports are mixed together with solenoid lengths of $5.0 \mathrm{~m}$ and respective fields of 10.2 T and 3.2 T. The flux of each $50 \mathrm{MeV}$ solenoid is returned in a channel around the beam pipe. The $500 \mathrm{MeV}$ solenoids alternate in polarity and are positioned symmetrically to minimize interference among their fringe fields, possibly with soft iron yokes to aid with field symmetrization. This can reduce harmful field components to less than 100 Gauss, which would then be nulled with special multipole windings around the solenoid ends.

Each beam has initial radius of $5.0 \mathrm{~cm}$ entering the final focus $(10.0 \mathrm{~m}$ from the target) and leaves with $3.25 \mathrm{~cm}$ radius and 0.0065 radian convergence half angle. An assumed emittance $\varepsilon_{\mathrm{n}}=3.75 \times 10-7 \mathrm{~m}$ produces final focus spot radius $\mathrm{a}_{\mathrm{s}}=\varepsilon_{\mathrm{n}} /(\beta \theta)=0.53 \mathrm{~mm}$ for the $500 \mathrm{MeV}$ beams and $1.7 \mathrm{~mm}$ for the $50 \mathrm{MeV}$ beams. Momentum spread increases this; For example, $\Delta p / p \leq \pm .003$ would contribute a diffuse spot edge $(0.43 \mathrm{~mm})$ in quadrature. The total is $\left(0.53^{2}\right.$ $\left.+0.43^{2}\right)^{1 / 2}=0.68 \mathrm{~mm}$.

A high degree of neutralization is required. For instance, the unshielded beam field is $>10 \mathrm{MV} / \mathrm{cm}$ near the target. The $H_{2}$ plasma introduced into the final compression beam lines and final focus strips and scatters the $R b^{+}$ions to a minor degree. With $\mathrm{n}_{\mathrm{H}_{2}}=10 \mathrm{n}_{\mathrm{b}}$ only about $0.1 \%$ of the $500 \mathrm{MeV}$ ions are lost to stripping and $1 \%$ of the $50 \mathrm{MeV}$ ions are lost (Bethe's formula). If pressure is higher than $10^{-3}-10^{-2}$ Torr $\left(3.5-35 \times 10^{13}\right.$ molecules $\left./ \mathrm{cm}^{3}\right)$ then a rough estimate indicates $R b$ will strip to about charge state +9 . Such ions scatter from $L i$ and also the heavier $\mathrm{Fl}$ and $\mathrm{Be}$ if they are present.

Scattering on the gas and/or plasma in the target chamber can be an important effect at the low kinetic energies considered in this paper. We did a number of Monte Carlo simulations to investigate how much scattering contributes to the focal spot size. In order to minimize the

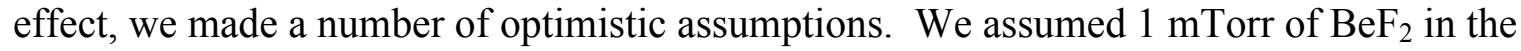
chamber compared to the 3 mTorr of FLiBe assumed in the RPD. We considered only the scattering on Fluorine, considered to be un-ionized. We also ignored stripping of the Rubidium beam and any scattering that occurs before the beam enters the chamber. (Beam stripping was a significant effect in the RPD design.) A scatter plot of position at the focal plane for the $50 \mathrm{MeV}$ beams is shown in Fig. 4(a). Without scattering all ions would hit the center. The fraction of particles falling inside a given radius is shown in Fig. 4(b). With scattering, $82 \%$ fall within $\mathrm{R}=$ $1 \mathrm{~mm}$. 


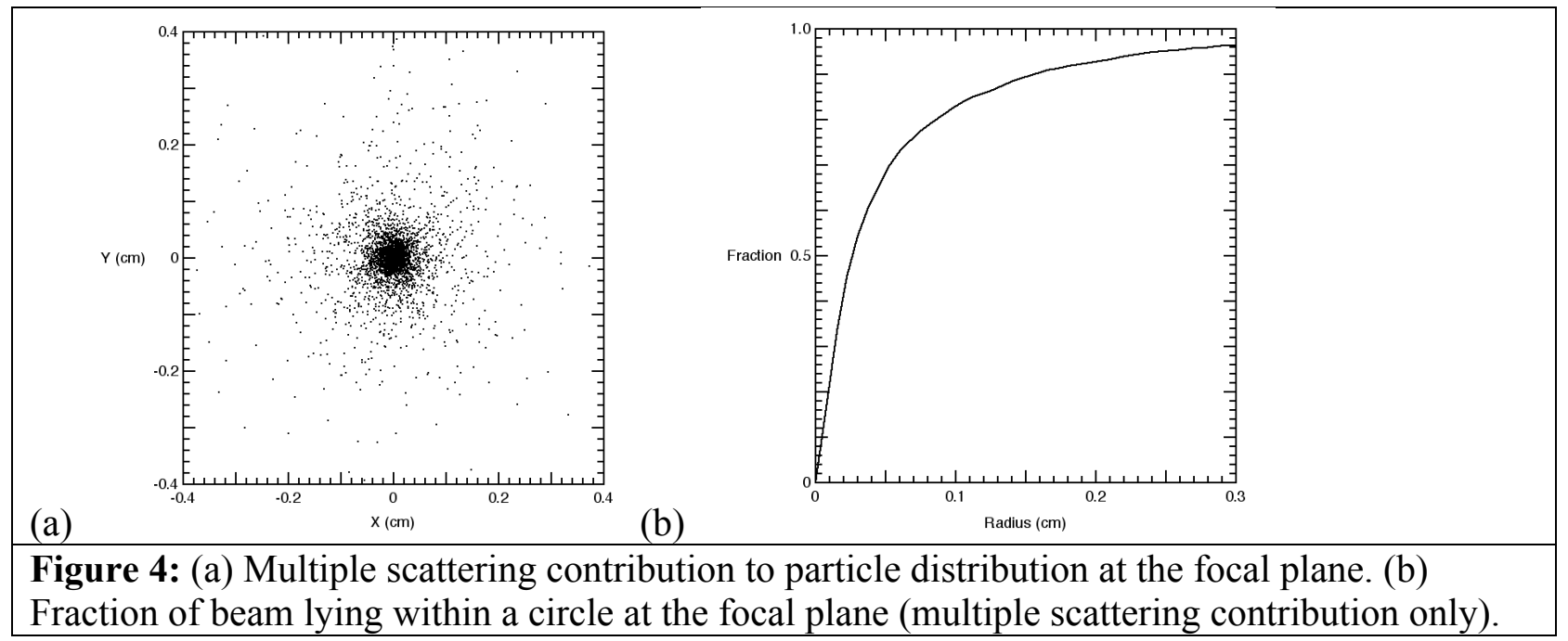

If we include the effects of injector emittance (Fig. 5), and assuming no emittance growth in accelerator, and ignoring other effects (such as aberrations, stripping, and imperfect neutralization) about half the $\mathrm{Rb}$ ions fall within an acceptable radius -- even with these optimistic assumptions.

(a)

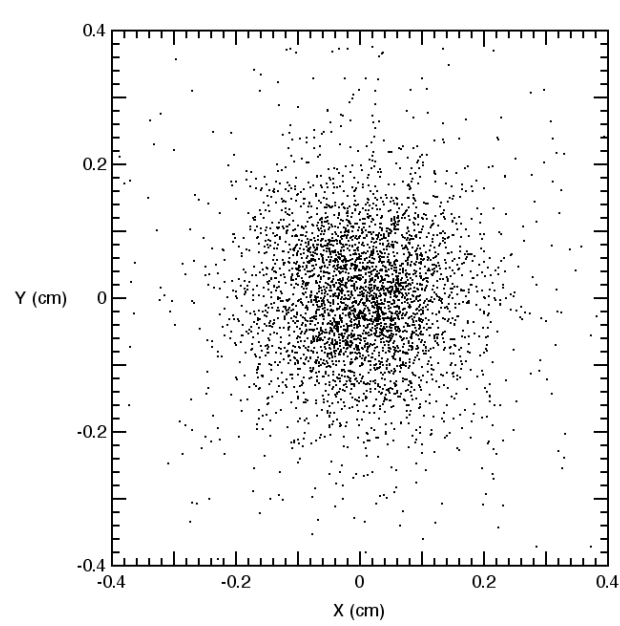

(b)

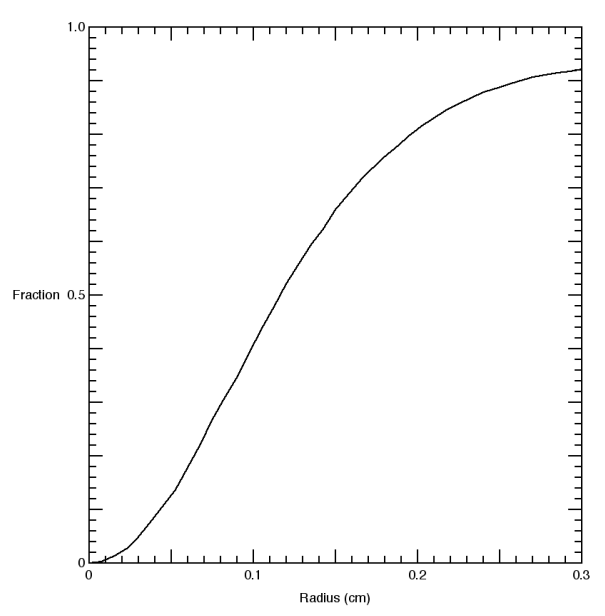

Figure 5: (a) Multiple scattering and emittance contribution to particle distribution at the focal plane. (b) Fraction of beam lying within a circle at the focal plane.

Above, we have used the injector emittance in our estimates of beam spot size on the fusion target, leading to an overly optimistic beam radius at the target. We are aware of numerous sources of longitudinal and transverse emittance growth. Since there is considerable uncertainty in estimates of emittance growth in drivers, target and driver designs often allow for a significant amount of growth from unspecified mechanisms and imperfections to make the design robust against the uncertainties. 
For example, nonlinear quadrupole fields, centroid oscillations, envelope mismatch (due to quadrupole gradient errors and energy variation, or natural tilt) will all contribute to emittance growth. The nonlinear field components, $\left(E \propto r^{n}, n>1\right)$ are a steep function of radius within the quadrupole bore, so their impact on emittance growth is enhanced by mismatch and centroid oscillations. Also, nonlinear fringe fields at the beginning and end of each quadrupole arise from the asymmetry of the interdigitated electrodes, and can be calculated from 3D models of the boundary conditions. For example, in the RPD, the injector emittance is $\varepsilon_{n i}=3.2 \times 10^{-7} \mathrm{~m} \cdot \mathrm{r}$ and the final emittance was estimated to be $8.6 \times 10^{-7} \mathrm{~m} \cdot \mathrm{r}$.

Using the emittance growth model in [Le-99], the estimated contribution from nonlinear fields in the " $2 \mathrm{D}$ " fields of the electrostatic quadrupoles is approximately $1.05 \cdot \varepsilon_{\mathrm{ni}}$, or a $5 \%$ increase in the emittance for an average beam radius $\overline{\mathrm{a}}=1.2 \mathrm{~cm}$ and a total of $\approx 2400$ quadrupoles per beamline. However, this does not include effects of centroid oscillations and beam envelope mismatch, which force particles to sample fields at a larger radius, justifying a larger effective $\overline{\mathrm{a}}$. If, for example, $\overline{\mathrm{a}}=2.0 \mathrm{~cm}$ within the $\mathrm{R}=3 \mathrm{~cm}$ bore, then this estimate of the final emittance, added in quadrature to the injector emittance would be:

$\varepsilon_{\mathrm{nf}}=\left(\varepsilon_{\mathrm{ni}}{ }^{2}+\left(\Delta \varepsilon_{\mathrm{n}}\right)^{2}\right)=\left(\left(3.75 \times 10^{-7}\right)^{2}+\left(3.39 \times 10^{-7}\right)^{2}\right)=4.7 \times 10^{-7} \mathrm{~m} \cdot \mathrm{r}=1.35 \cdot \varepsilon_{\mathrm{ni}}$

This may also be an underestimate, since there will be additional emittance growth from the nonlinear fringe fields, growth in the drift compression quadrupoles and transport lines, and growth in the final focus section. In this context, an overall margin of $\varepsilon_{\mathrm{nf}} \approx(2-3) \cdot \varepsilon_{\mathrm{ni}}$ may be reasonable.

\section{Issues and Observations}

In this section we list a number of issues to help guide the next iterations in direct drive target and driver design. Some are fundamental to the constraints set by the target design in Sections 1 and Fig. 1. Others arise from the driver design choices made in the subsequent sections. Note that the driver design is far from optimized. Though we attempted to resolve internal inconsistencies between design choices and known physics or technology, some remained unresolved in the interest of a timely completion of this report:

A. The overall driver length $(\sim 1.6 \mathrm{~km})$, short compared to other HIF driver designs, is an attractive feature. This is helpful when considering site possibilities for an ETF or fullscale power plants.

B. The choice of electrostatic focusing in the accelerator avoids uncertainties and possible emittance increase due to electron-cloud effects. This removes the urgency for some R\&D from the development path. In previous HIF driver designs electrostatic focusing has been used in the front end of a driver (e.g., $\mathrm{E}<50 \mathrm{MeV}$ ), followed by magnetic focusing for most of the accelerator [Me-98].

C. The proposed all-electrostatic focusing in the accelerator is unprecedented in length and number of HV electrostatic components. Questions to be explored include: What is a tolerable mean time between failures? What are the significant failure mechanisms (e.g.: 
beam halo induced discharges; UV induced discharges from electrode imperfections)? Significant R\&D development would be required for a high confidence design.

D. The packing of 904 beams at the reactor chamber wall $(R=5 \mathrm{~m})$ is complicated and uses $27 \%$ of $4 \pi$ steradians in two bundles each with half-angle of $42^{\circ}$. This is large compared to the Robust Point Design (RPD) for an indirect drive (hohlraum) target [Yu-03], where the RPD beam bundle fit in a cone half-angle of 24 degrees, or $8 \%$ of $4 \pi$ steradians. The number of beams is unlikely to lead to a design with an attractive cost for the drift compression section. Technical feasibility of the drift compression section is also a concern.

E. Among the beam groups, the required longitudinal phase space density for the $(50 \mathrm{MeV})$ 2.1-ns and 0.8-ns shock $(8.53<\mathrm{t}<10.615 \mathrm{~ns}$ and $10.865<\mathrm{t}<11.665 \mathrm{~ns}$ in Fig. 2, beam groups $\mathrm{B}$ and $\mathrm{C}$ ) are most challenging. The assumed bunch length may be incompatible with achieving the required beam conditions at the fusion target. Assuming a voltage waveform accuracy at the injector of $\pm 0.1 \%$, the initial longitudinal phase space is 0.021 $\mathrm{eV} \cdot \mathrm{sec}$. The resulting random energy spread at the target is $\pm 2.7 \%, \pm 10.2 \%, \pm 26.7 \%$ and $\pm 1.2 \%$ for beam groups A-D respectively. The bunch duration of beams in group $\mathrm{C}$ at the exit of the final focus magnet is $\approx 9 \mathrm{~ns}$. Thus, the energy spread (due to injector emittance) for beams of group $\mathrm{C}$ at the exit of the final focusing solenoid is $4.5 \%$ (due to reduced tilt to 0.012 ). This neglects the additional longitudinal emittance growth through the accelerator due to acceleration waveform imperfections and other effects. Reducing the initial bunch duration can mitigate this problem, which leads to an increase in the number of beams.

F. There are numerous open questions concerning the physics design and engineering of the time varying electric quadrupoles (Sec. 5) designed to compensate for the chromatic aberration from the drift compression tilt.

G. Path-length imperfections for different beams will likely require time-of flight correction by varying the acceleration among different beams, or variable bends in the drift compression section.

H. To avoid asymmetries in low order modes $(l=1$ to 4$)$ in the beam irradiation on the target, the beam pointing accuracy of the main pulse (360 beams, $500 \mathrm{MeV}$ ) may need to be $\approx 30 \mu \mathrm{m}$ or less. Accuracies of this level have been considered very difficult.

I. There is little or no allowance for transverse emittance growth in the accelerator. We are making a very optimistic assumption here by using only the injector emittance for calculating the final focus beam size. For the 50 and $500-\mathrm{MeV}$ beams, the emittancelimited focal spot radii $\mathrm{R}_{\mathrm{tgt}}$ are $1.7 \mathrm{~mm}$ and $0.53 \mathrm{~mm}$ respectively, assuming an injector normalized emittance of $3.75 \times 10^{-7} \mathrm{~m}$ and a final convergence angle $\theta=6.5 \mathrm{mrad}$. The target capsule radius is $R_{\text {cap }}=1.2 \mathrm{~mm}$. The value of $R_{\text {tgt }}=0.53 \mathrm{~mm}$ is large compared to the requirement derived by Runge and Logan for polar direct drive [Ru-09]: the beam radius should be $0.3-0.4 \mathrm{R}_{\text {cap }}$, assuming 100 beams though this would be relaxed with the larger number of beams here. 
J. Plasma instabilities and neutron dumps are known issues for heavy ion fusion drivers. We did not study these for this report because they cannot ameliorate other issues and we were already unable to find an acceptable solution to the driver constraints.

$\mathrm{K}$. We have assumed perfect neutralization, but there are strong theoretical reasons to believe this does not happen. In the limit of steady electron flow, a moderate strength pinch force has recently been computed [Do-09].

L. Finally, an open question is the scaling of this technical approach to a power plant. For example, questions to address are whether a power plant would use sufficiently similar ion injectors, accelerator, drift compression, and neutralization designs.

This target design reduces the required beam energy from 6.7 MJ (RPD target design, [Ca-00]) to $<0.5 \mathrm{MJ}$, while making the accelerator more challenging than the RPD and other indirect drive, higher driver energy designs. Target designs with higher values of ion kinetic energy, and intermediate values of driver energy will be considered next.

\section{REFERENCES:}

[Ba-05] J.J. Barnard et al., Nucl. Inst. Meth. A 544 (2005) 243.

[Bi-05] F.M. Bieniosek et al., Phys. Rev. PRST-AB 8, 010101 (2005).

[Ca-00] D.A. Callahan-Miller, Phys. Plasmas, 7, No. 5, (2000) 2083.

[Do-09] M. Dorf et al., Phys. Rev. Lett., 103, 075003 (2009). Also, I. Kaganovich et al., submitted to Physics of Plasmas, (2010).

[Lo-08] B. G. Logan, L. J. Perkins, and J. J. Barnard, Phys. Plasmas 15, 072701 (2008).

[Fa-96] A. Faltens and P.A. Seidl, XVIIth International Symposium on Discharges and Electrical Insulation in Vacuum, 1996. Berkeley, CA, USA. ISBN: 0-7803-2906-6.

[Fe-89] T.J. Fessenden et al., Nucl. Inst. Meth. A278 (1989) 13. M.G . Tiefenback and D. Keefe, IEEE Trans. Nucl. Sci. NS-32 (1985) 2483.

[Ho-88] J. Hovingh et al., Fusion Technology 13, 255 (1988).

[Le-99] E.P. Lee, Rough Estimate Of Emittance Growth From Magnetic Field Errors, (1999) HIFAR Note \#486.

[Lo-09] See slide \#7 of "Overview of Fusion Program at LBNL" G. Logan http://fire.pppl.gov/fpa09 HIF Logan.pdf.

[Me-98] W.R. Meier et al. Nucl. Inst. Meth A415 249 (1998).

[Pr-05] L.R. Prost et al., Phys. Rev. PRST-AB 8, 020101 (2005).

[Ru-09] J. Runge and B. G. Logan, PHYSICS OF PLASMAS 16, 033109 (2009).

[Se-03] P.A. Seidl et al., Phys. Rev. PRST-AB, 6, 090101 (2003).

[Sn-02] Presentations and papers presented at the Snowmass 2002 Fusion Energy Sciences Summer Study, 8-19 July 2002. See for example, http://web.gat.com/conferences/snowmass/working/ife/driver/d2/ibx-white-paper.html

[Yu-03] S. Yu et al., Fusion Sci. Tech. 44, 2329 (2003). 